\section{A Rapid Predictive System for Pancreatic Fistula after Pancreaticoduodenectomy: A Report from a Low-Volume Center}

\section{Abstract}

Background: Postoperative pancreatic fistula (POPF) is a serious complication of pancreaticoduodenectomy (PD).

Methods: We retrospectively reviewed the records of 109 consecutive patients who underwent PD between January 2006 and September 2015 in Saitama City Hospital. Preoperative and intraoperative variables were evaluated by univariate and multivariate logistic regression analyses to establish an immediate prediction model for POPF.

Results: POPF grade A developed in 13 patients [12\% (13/109)], grade B in 19 [17\% (17/109)], and grade $C$ in 3 [3\% (3/109)]. Multivariate analysis demonstrated that the diameter of the main pancreatic duct (dMPD) $\leq 3.2 \mathrm{~mm}$ [odds ratio (OR) 4.26, 95\% confidence intervals ( $\mathrm{Cls}$ ) $1.45-12.50, \mathrm{P}=0.008$ ], and operative times $\geq$ 425 min (OR 10.20, 95\% Cls 2.67-39.00, P<0.001) were significant independent risk factors for POPF after PD. Using a combination of these two factors, the 109 patients were divided into two groups, and the incidence of POPF in patients with $\mathrm{dMPD} \leq 3.2 \mathrm{~mm}$ and with operative times $\geq 425$ min was significantly higher than in those without them both [62\% (26/42) versus $13 \%(9 / 67), P<0.001]$. The predictive accuracy, sensitivity, specificity, positive predictive value, and negative predictive value were $77 \%(84 / 109), 74 \%(26 / 35), 78 \%(58 / 74), 62 \%(26 / 42)$, and $87 \%$ (58/67), respectively.

Conclusions: Our predictive model of POPF after PD has high accuracy. The immediate ability to predict POPF after PD using our model results in better postoperative management of patients undergoing PD.

Keywords: Postoperative pancreatic fistula; Pancreaticoduodenectomy; Predictive model

\section{Kaoru Takeshima, Kazuo Yamafuji and Kiyoshi Kubochi}

\author{
Department of Surgery, Saitama City \\ Hospital, Saitama, Japan
}

Corresponding author: Kaoru Takeshima

”pordoc1964@yahoo.co.jp

Department of Surgery, Saitama City Hospital, Saitama, 2460 Mimuro, Midori-ku, Saitama, 336-0911, Japan.

Tel: $+81-488734111$

Fax: $+81-488737982$

Citation: Takeshima K, Yamafuji K, Kubochi K. A Rapid Predictive System for Pancreatic Fistula after Pancreaticoduodenectomy: A Report from a Low-Volume Center. J Univer Surg. 2017, 5:2.

\section{Introduction}

Postoperative pancreatic fistula (POPF) is one of the most severe postoperative complications of pancreaticoduodenectomy (PD). This complication, occurring in $2 \%$ to $20 \%$ of patients after PD, often leads to miserable consequences, such as abscess formation, sepsis, and abdominal hemorrhage. These events result in longer hospital stays, increased hospital costs, and increased post-operative deaths after PD [1,2]. If the development of POPF after PD due to preoperative and intraoperative factors could be predicted immediately, surgeons could better manage it, resulting in improved morbidity, mortality, and economic losses associated with PD. Perioperative risk or predictive factors, including pancreatic texture, pancreatic duct diameter, anastomosis type, pathology, and blood loss, correlated with POPF and evaluated by multivariate analysis have been described in the literature [2-5]. These factors are very useful for the prediction of POPF. If POPF could be predicted by the time the operation was finished, its postoperative treatment could be initiated as soon as possible. The purpose of this study was to analyze whether it was possible to immediately predict POPF after PD by evaluation of preoperative and intraoperative factors. 


\section{Patients and Methods}

\section{Study design}

The records of 112 consecutive patients who underwent PD between January 2006 and September 2015 for periampullary pathologies, including pancreatic head carcinoma, distal bile duct carcinoma, ampulla of Vater carcinoma, duodenal carcinoma and others, were retrospectively reviewed. Two patients who underwent PD and then died from complications other than POPF within the $8^{\text {th }}$ postoperative day, and one patient who underwent total pancreatectomy, were excluded. The Ethics Review Board of Saitama City Hospital approved this study including the use of the opt-out consent procedure (the approval code; A2714).

\section{Preoperative assessment}

The preoperative diagnosis was performed by clinical assessment (age, gender, symptoms, and signs), laboratory investigation (complete blood counts, creatinine, serum total bilirubin level, serum amylase level, and tumor markers such as carcinoembryonic antigen and carbohydrate antigen 19-9), and multidetector-row computed tomography (MDCT). MDCT was performed immediately upon admission to Saitama City Hospital. The diameter of the main pancreatic duct (dMPD) right above the portal vein was measured in the axial view of the MDCT. Endoscopic retrograde cholangiopancreatography (ERCP) was performed in all patients. The biliary drainage of patients with obstructive jaundice was examined using an endoscopic biliary stent during ERCP.

\section{Surgical procedures}

All operations were performed by three experienced surgeons or under their supervision. The total of ten surgeons including those three experienced surgeons operated on the patients and the procedures included conventional PD in 56 patients, pyloruspreserving PD in six patients, and subtotal stomach-preserving PD in 47 patients. Combined portal vein resection was performed in ten patients. The type of digestive tract reconstruction was the Child's modified method in 105 cases and Imanaga's method [6] in four cases. In all cases, pancreaticojejunostomy was performed by end-to-side and duct-to-mucosa anastomosis. No pancreaticogastrostomies were performed. In all patients, the duct-to-mucosa anastomosis was performed with a double layer anastomosis using interrupted 6-0 non-absorbable braided suture (Ethibond Extra, Ethicon, Inc.) or interrupted 5-0 absorbable suture (PDS*II, Ethicon, Inc.). In the double layer suture, the pancreatic parenchyma was sutured to the jejunal wall by 3-0 silk-braided suture (Perma-Hand Silk, Ethicon, Inc.) or 4-0 absorbable-braided suture (Coated VICRYL, Ethicon, Inc.). A 5- or 6-French (Fr) polyvinyl chloride tube (Sumitomo Bakelite, Tokyo, Japan) was inserted into the pancreatic duct as an internal stent in all cases. Omental wrapping of major vessels was performed in 105 cases and fibrin glue (Bolheal, Teijin Pharma Limited, Tokyo, Japan) was employed in four patients. The pancreaticojejunostomies and choledocojejunostmies were drained routinely. In the 11 patients before January 2007, the pancreaticojejunostomy drainage was performed using a 12-
Fr Penrose drain inserting stomach tube (Salem Sump Tube, Nippon Covidien Ltd, Shizuoka, Japan) and the drainage fluid was continuously suctioned. After that date, closed silicone drains (Blake Silicone Drain, Ethicon Inc.) were used for both pancreaticojejunostomies and choledocojejunostmies.

\section{Postoperative management}

All patients received prophylactic antibiotics intra-operatively and 3 days to 4 days postoperatively. Prophylactic octreotide was not routinely used. Output volumes from intraoperatively placed drains were recorded daily until the drain was removed. The amylase level of the drainage fluid was measured on postoperative day (POD) 3, whereas in some cases, it was measured on POD 1 or 2 at the discretion of the surgeon because of clinical limitations. In patients without POPF, the drains were removed before the $8^{\text {th }} P O D$. The drains were maintained longer in patients with POPF. The nasogastric tube was typically removed at $P O D 1$, but in six patients who had pylorus-preserving $P D$, the nasogastric tube was removed between the $6^{\text {th }}$ and $8^{\text {th }}$ POD. The patients resumed meals with a fluid diet followed by a regular diet when they passed flatus.

\section{Definition of POPF}

In Saitama City Hospital, POPF was diagnosed using the International Study Group for Pancreatic Fistula (ISGPF) guidelines [1]. According to these guidelines, POPF is defined as any measurable volume of draining fluid on or after POD 3 with amylase content greater than three times the upper normal serum activity from an intraoperatively placed drain. Furthermore, the POPF was graded as A, B, or C by nine clinical criteria of the ISGPF guidelines. POPF Grade A is a so-called "transient fistula" and has no impact on the clinical outcome. POPF Grade B has a clinical impact requiring several treatments such as persistent drainage and radiological intervention. POPF Grade $C$ is the most severe complication and results in sepsis, abdominal hemorrhage, or patient death. In this study, we evaluated drain amylase levels on POD 3; however, in some cases, the evaluation was conducted on POD 1 or 2 at the discretion of the surgeon because of clinical limitations. Patients were considered not to have POPF when the following were noted in the medical records: the drains were removed before POD 8; without measurement of the amylase level of drain fluid; and there was a good postoperative clinical course. Patients were diagnosed as POPF grade B when the fluid appearance of the drain next to the pancreaticojejunostomy was greenish, brown, or infectious without a high amylase content of the drain output.

\section{Data collection}

The aim of this study was to determine whether the rapid prediction of POPF by preoperative and intraoperative variables was possible. Therefore, only preoperative and intraoperative data were collected from all patients' baseline history and physical examination records. Preoperative variables included patients' demographics (age and gender), symptoms (diabetes mellitus), characteristics [body mass index (BMI)], laboratory exams [serum albumin, total bilirubin at admission and on the 
preoperative day, serum creatinine, and tumor markers including carcinoembryonic antigen (CEA) and carbohydrate antigen 19-9 (CA19-9)], and preoperative dMPD by MDCT. Intraoperative variables included operative time, amount of blood loss, and need for a blood transfusion.

\section{Statistical analysis}

Statistical analyses were performed with $\mathrm{R}$ commander (version 2.1.7) based on $R$ (version 3.2.0) and with EZR, which is a modified version of $\mathrm{R}$ commander [7]. The $\mathrm{R}$ is free to download and install from the Comprehensive R Archive Network (http:// cran.r-project.org/). The normality of continuous valiables examined using the Shapiro-Wilk test. Continuous variables, including albumin and creatinine, which followed normal distributions, were described as mean \pm standard deviation and were examined by the independent two-sample $t$-test. The other continuous variables, which did not follow normal distributions, were expressed as median values with interquartile range and evaluated by the Wilcoxon rank sum test. Categorical variables were analyzed by Pearson's chi-squared test or Fisher's exact test. $P$ values $<0.050$ were considered to be statistically significant. Variables with $P<0.100$ by univariate analysis were entered into a logistic regression analysis. Receiver operating characteristics (ROC) analyses were performed to determine the cut-off values using Youden index. Continuous variables were converted into binary variables based on cut-off values. The results of logistic regression analysis were described as odds ratios (OR) with $95 \%$ confidence intervals $(95 \% \mathrm{Cls})$. The estimation of the prediction of POPF was performed by the predictive accuracy, sensitivity, specificity, and positive and negative predictive value (PPV and NPV) calculated on the basis of the incidence of POPF in the two groups into which the patients were divided by using a combination of the significant risk factors.

\section{Results}

Histological examination revealed the diagnosis in 94 in the 109 patients; pancreatic head carcinoma $(n=41)$, distal bile duct carcinoma $(n=30)$, ampulla of Vater carcinoma $(n=20)$ and duodenal carcinoma $(n=3)$. The diagnosis in the other 15 patients was duodenal gastrointestinal tumor in 2, cystic duct carcinoma in 1 , metastatic pancreatic head carcinoma in 1 , colon carcinoma invading to the dudenum in 1 , intraductal papillary mucinous neoplasm in 4, mucinous cystic tumor in 1, neuroendcrine tumor in 1 and benign disorders in 4 . The administration of prophylactic antimicrobial agents is shown in (Table 1). In a total of 109 patients, the overall incidence of POPF was 35/109 (32\%). The patients with grades A, B, and C POPF were $13[12 \%$ (13/109)], 19 [17\% (19/109)], and 3 [3\% (3/109)], respectively. All grade $C$ patients experienced abdominal hemorrhage from a pseudoaneurysm of the gastroduodenal artery stump or the common hepatic artery and underwent radiological intervention. No patients died from POPF in Saitama City Hospital.

The preoperative and intraoperative variables that were evaluated by univariate analysis are shown in (Table 2). The univariate analysis was performed using nine preoperative variables and three intraoperative variables. There was a significant difference between the median IMPD of patients with POPF and without POPF $(P=0.004)$. The operative times of patients with POPF were significantly longer than those of patients without POPF $(P=0.044)$. The univariate analysis demonstrated that serum level of total bilirubin at admission and CA19-9 were not statistically significant but tended to be lower in patients with POPF. A ROC curves demonstrated that the cut-off values of 4 valiables with $P<0.100$, dMPD, operative time, total bilirubin at admission and CA19-9 were $3.2 \mathrm{~mm}, 425 \mathrm{~min}, 7.5 \mathrm{mg} / \mathrm{dl}$ and $46.4 \mathrm{U} / \mathrm{ml}$, respectively, of which the area under the curve (AUC) were $0.671,0.620,0.604$ and 0.607 , respectively. These results are shown in (Table 3).

These four valiables were further evaluated using multivariate analysis. (Table 4) shows the results of the logistic regression analysis. Both dMPD $\leq 3.2 \mathrm{~mm}$ and operative times $\geq 425 \mathrm{~min}$ were significant independent risk factors associated with the incidence of POPF ( $P=0.008$ and $<0.001$, respectively) and the OR and $95 \% \mathrm{Cls}$ of each was 4.26 and 10.20 , and $1.45-12.50$ and 2.67-39.00, respectively.

The 109 patients in this study were divided into two groups using the combination of $\mathrm{dMPD}$ and operative time. Group A comprised patients with dMPD $\leq 3.2 \mathrm{~mm}$ and operative time's $\geq 425 \mathrm{~min}$. Group B comprised those without dMPD $\leq 3.2 \mathrm{~mm}$ or without operative time's $\geq 425 \mathrm{~min}$. The incidence of POPF in groups $A$ and $B$ were $62 \%(26 / 42)$ and $13 \%(9 / 67)(P<0.001)$, respectively (Table 5), and the predictive accuracy, sensitivity, specificity, PPV, and NPV were 77\% (84/109), 74\% (26/35), 78\% (58/74), 62\% (26/42), and 87\% (58/67), respectively (Table 6).

\section{Discussion}

POPF after PD is one of the most important complications correlating to patient death with adverse effects on the medical economy. In spite of advancements in surgical techniques and perioperative patient care, POPF develops in $2 \%$ to $20 \%$ of patients undergoing PD [1]. This suggests that the prevention of POPF is very difficult. Therefore, improved postoperative management of POPF after PD could lead to reduced mortality and financial savings. The immediate prediction of POPF after $\mathrm{PD}$ is necessary to rapidly institute the treatment of POPF with better outcomes.

A great number of studies have described the risk factors for POPF after PD. Age, gender, diabetes mellitus, diameter of the main pancreatic duct, pancreatic texture, pathological diagnosis, amount of blood loss, and operative time are independent risk factors in multivariate analyses [3,8-14]. It has been shown

Table 1: The administration of prophylactic antimicrobial agents.

\begin{tabular}{|cc|}
\hline Agent & No. (\%) \\
\hline Sulbactam/Cefoperazone & $53(53)$ \\
\hline Cefmetazole & $35(32)$ \\
\hline Flomoxef & $8(7)$ \\
Cefazolin & $3(3)$ \\
Others* & $5(5)$ \\
*Ceftadizime $(n=1)$ Cefepime $(n=1)$ Piperacillin $(n=1)$ Amikacin $(n=1)$ \\
Vancomycin $(n=1)$
\end{tabular}


Table 2: Univariate analysis of preoperative and intraoperative variables (n\%).

\begin{tabular}{|c|c|c|c|}
\hline Variables & POPF (N=35) & No POPF (N=74) & $P$ value \\
\hline \multicolumn{4}{|l|}{ Preoperative } \\
\hline Age (year) & $70(62.5-73.5)$ & $69(61.5-74.8)$ & 0.966 \\
\hline \multicolumn{4}{|l|}{ Gender } \\
\hline Male & $22(63)$ & $45(61)$ & 1 \\
\hline Female & $13(37)$ & $29(39)$ & \\
\hline \multicolumn{4}{|l|}{ Diabetes mellitus } \\
\hline Yes & $10(29)$ & $23(31)$ & 0.966 \\
\hline No & $25(71)$ & $51(69)$ & \\
\hline $\mathrm{BMI}, \mathrm{kg} / \mathrm{m}^{2}$ & $22.5(20.8-24.5)$ & $21.3(19.2-23.2)$ & 0.106 \\
\hline $\begin{array}{l}\text { Serum albumin, } \mathrm{mg} / \mathrm{dl} \text {, bilirubin, } \\
\mathrm{mg} / \mathrm{dl}\end{array}$ & $3.7 \pm 0.5$ & $3.6 \pm 0.5$ & $0.182^{+}$ \\
\hline At admission & $1.5(0.8-6.6)$ & $3.4(0.9-11.3)$ & 0.081 \\
\hline On the preoperative day & $0.8(0.6-1.2)$ & $1.0(0.7-1.6)$ & 0.113 \\
\hline Serum creatinine, $\mathrm{mg} / \mathrm{dl}$ & $0.72 \pm 0.17$ & $0.72 \pm 0.19$ & $0.955^{+}$ \\
\hline \multicolumn{4}{|l|}{ Tumor marker } \\
\hline CEA, ng/ml & $2.7(1.7-3.7$ & $2.4(1.7-3.7)$ & 0.733 \\
\hline CA19-9, U/ml & $26.1(11.9-75.8)$ & $54.7(19.9-233)$ & 0.071 \\
\hline $\mathrm{dMPD}, \mathrm{mm}$ & $2.5(1.8-3.1)$ & $3.5(2.2-5.9)$ & 0.004 \\
\hline \multicolumn{4}{|l|}{ Intraoperative } \\
\hline Operative time, $\min$ & $487(451-542)$ & $457(391-520)$ & 0.044 \\
\hline Blood loss, $\mathrm{ml}$ & $885(668-1288)$ & $881(560-1229)$ & 0.448 \\
\hline \multicolumn{4}{|l|}{ Transfusion } \\
\hline Yes & $3(9)$ & $10(16)$ & $0.543^{++}$ \\
\hline No & $32(91)$ & $64(84)$ & \\
\hline \multicolumn{4}{|c|}{$\begin{array}{l}\text { Values are expressed as median (interquartile range) or mean } \pm \text { standard deviation. } \\
\text { BMI body mass index, CEA carcinoembryonic antigen, CA19-9 carbohydrate antigen 19-9, dMPD the diameter of the main pancreatic duct } \\
+ \text { independent two-sample } t \text { test } \\
++ \text { Fisher's exact test }\end{array}$} \\
\hline
\end{tabular}

Table 3: $A$ ROC analysis of valiables with $P<0.100$ by univariate analysis.

\begin{tabular}{|c|c|c|c|c|}
\hline Valiables & cut-off value & AUC & Sensitivity & Specificity \\
\hline $\mathrm{dMPD}, \mathrm{mm}$ & 3.2 & 0.671 & 0.8 & 0.554 \\
\hline Operative time, $\min$ & 425 & 0.62 & 0.914 & 0.419 \\
\hline Total bilirubin at admission, $\mathrm{mg} / \mathrm{dl}$ & 7.5 & 0.604 & 0.857 & 0.365 \\
\hline
\end{tabular}

Table 4: Multivariate logistic regression analysis.

\begin{tabular}{|c|c|c|c|}
\hline Variables & OR & $95 \% \mathrm{Cls}$ & $P$ value \\
\hline \multicolumn{4}{|l|}{$\mathrm{dMPD}, \mathrm{mm}$} \\
\hline$\leq 3.2$ vs. $>3.2$ & 4.26 & $1.45-12.50$ & 0.008 \\
\hline \multicolumn{4}{|c|}{ Operative time, $\min$} \\
\hline$\geq 425$ vs. $<425$ & 10.2 & $2.67-39.00$ & $<0.001$ \\
\hline \multicolumn{4}{|c|}{ Total bilirubin at admission, $\mathrm{mg} / \mathrm{dl}$} \\
\hline$\leq 7.5$ vs. $>7.5$ & 2.22 & $0.59-8.38$ & 0.238 \\
\hline \multicolumn{4}{|l|}{ CA19-9, U/ml } \\
\hline$\leq 46.4$ vs. $>46.4$ & 1.85 & $0.60-5.74$ & 0.286 \\
\hline
\end{tabular}

that the occurrence of POPF seems to be intricately associated with preoperative, intraoperative, and postoperative variables. We assessed the preoperative and intraoperative variables in univariate and multivariate analyses in order to predict POPF immediately after PD.
We revealed that the IMPD and operative time were statistically significant risk factors in a logistic regression analysis. Our study showed that POPF developed more frequently in patients with $\mathrm{dMPD} \leq 3.2 \mathrm{~mm}$ than in those with $\mathrm{dMPD}>3.2 \mathrm{~mm}$. Previous reports, including prospective or retrospective studies, were 
Table 5: The incidence of POPF in group A and group B ( $n \%)$.

$\begin{array}{cccc}\text { Group } & \text { POPF (N=35) } & \text { no POPF (N=74) } & P \text { value } \\ \text { Group A } & 26(62) & 16(38) & <0.001 \\ \text { Group B } & 9(13) & 58(87) & \\ \text { Group A; patients with dMPD } \leq 3.2 & \text { mm and operative times } \geq 425 \text { min } \\ \text { Group B; patients without dMPD } \leq 3.2 & \text { mm or without operative time } \\ \geq 425 \text { min }\end{array}$

Table 6: The estimation of the prediction for POPF.

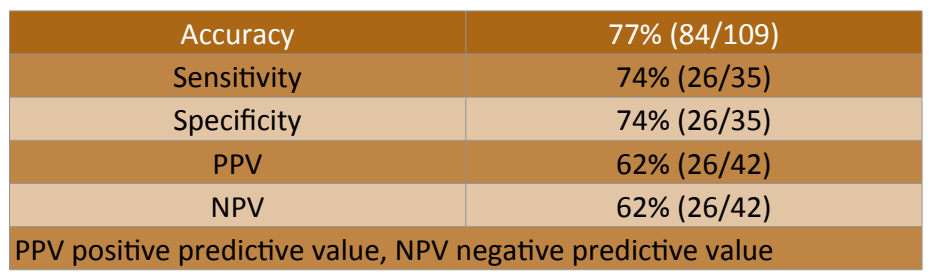

consistent with our findings and described that IMPD was a significant risk factor for POPF after PD $[11,15]$. Furthermore, we showed that there was a statistically significant correlation between the operative time and incidence of POPF. The patients with operative time's $\geq 425$ min developed POPF approximately 10 times as frequently as those with operative times $<425 \mathrm{~min}$. A previous study also described that the operative time was a significant risk factor based on a multivariate analysis [14].

Using the combination of only these two significant variables, dMPD and operative time, we developed a predictive method for POPF. The predictive accuracy of our method was high at $77 \%$. Our model had low sensitivity and high specificity. This means that POPF is very likely to develop in the patients diagnosed with POPF after PD by our model, but our model will not detect all cases of POPF after PD. It is possible for surgeons to better predict and manage POPF immediately after PD using our model. A recent study [16] revealed that intra-operative amylase concentration in peri-pancreatic fluid is an effective variable for a predictive modeling for POPF. This implies the possibility of refining our predictive model by utilizing intra-operative amylase concentration in peri-pancreaticojejunostomy fluid as a relevant predictor.

Our system predicts not only clinically relevant POPF but also POPF grade $A$. Some studies have reported predictive systems for POPF grades B or C [17-19], and were very useful; however, we think it is important to estimate all grades of POPF. There was a case where a drain that was inserted following a pancreaticojejunostomy was removed due to the absence of abnormal discharge; however, infectious discharge was later discovered, and the drain was reinserted with fistulography. We thus evaluated this patient as POPF Grade B. While this case is rare, this suggests that patients diagnosed with POPF Grade $A$ or no POPF could actually have POPF Grade B and we should therefore also predict POPF grade A. Several definitions of POPF after PD were proposed using drain output and/or amylase levels of drain fluid [20-23]; the ISGPF guidelines have become the gold standard for POPF diagnosis since 2005. However, as our previously mentioned case showed, it is essential to carefully observe the postoperative clinical course of patients undergoing PD even if they do not fulfill the ISGPF guidelines.

Though we revealed that our method had high accuracy for the prediction of POPF after PD, there are some weak points in our system. Our study is based on a small number of patients. Many reports about POPF after PD comprise a large number of patients and some studies have more than two hundred patients [3,5,13,17-19]. As our institute is not a high-volume center, we had difficulty accumulating patients undergoing PD. The biggest problem with our method is that we were unable to validate it. In a previous report that included a large number of cases undergoing PD, the cases were divided into two groups to develop a predictive system, which was then validated in another group [18]. As we showed in this report, it is essential to validate predictive models; however, it was difficult for us to validate our model because of the small number of cases that underwent PD in Saitama City Hospital. We understand that it is necessary to validate our model in the future with a prospective study based on further accumulation of patients undergoing PD.

\section{Conclusion}

In conclusion, we developed an immediate predictive system with high predictive accuracy for POPF of all grades after PD using a combination of two risk factors. Our model may be very useful for surgeons to better predict and subsequently rapidly treat POPF after PD. 


\section{References}

1 Bassi C, Dervenis C, Butturini G, Fingerhut A, Yeo C, et al. (2005) International Study Group on Pancreatic Fistula D. Postoperative pancreatic fistula: An international study group (isgpf) definition. Surgery 138: 8-13.

2 Akamatsu $N$, Sugawara $Y$, Komagome $M$, Shin $N$, Cho $N$, et al. (2010) Risk factors for postoperative pancreatic fistula after pancreaticoduodenectomy: The significance of the ratio of the main pancreatic duct to the pancreas body as a predictor of leakage. J Hepatobiliary Pancreat Sci 17: 322-328.

3 Pratt WB, Callery MP, Charles M, Vollmer Jr (2008) Risk prediction for development of pancreatic fistula using the isgpf classification scheme. World J Surg 32: 419-428.

4 Okabayashi T, Maeda H, Nishimori I, Sugimoto T, Ikeno T, et al. (2009) Pancreatic fistula formation after pancreaticooduodenectomy; For prevention of this deep surgical site infection after pancreatic surgery. Hepato-gastroenterology 56: 519-523.

5 Kawai M, Tani M, Hirono S, Ina S, Miyazawa M, et al. (2009) How do we predict the clinically relevant pancreatic fistula after pancreaticoduodenectomy? An analysis in 244 consecutive patients. World J Surg 33: 2670-2678.

6 Imanaga H (1960) A new method of pancreaticoduodenectomy designed to preserve liver and pancreatic function. Surgery 47: $577-$ 586. Kanda Y (2013) Investigation of the freely available easy-to-use software 'EZR' for medical statistics. Bone marrow transplantation 48: 452-458.

7 Kanda Y (2013) Investigation of the freely available easy-to-use software 'ezr' for medical statistics. Bone marrow transplantation 48: 452-458.

8 Riall TS, Reddy DM, Nealon WH, Goodwin JS (2008) The effect of age on short-term outcomes after pancreatic resection: A populationbased study. Annals of surgery 248: 459-467.

9 Kamoda Y, Fujino Y, Matsumoto I, Shinzeki M, Sakai T, et al. (2008) Usefulness of performing a pancreaticojejunostomy with an internal stent after a pancreatoduodenectomy. Surg Today 38: 524-528.

10 Satoi S, Takai S, Matsui Y, Terakawa N, Iwaki R, et al. (2006) Less morbidity after pancreaticoduodenectomy of patients with pancreatic cancer. Pancreas 33: 45-52.

11 Poon RT, Fan ST, Lo CM, Ng KK, Yuen WK, et al. (2007) External drainage of pancreatic duct with a stent to reduce leakage rate of pancreaticojejunostomy after pancreaticoduodenectomy: A prospective randomized trial. Annals of surgery 246: 425-435.
12 Lin JW, Cameron JL, Yeo CJ, Riall TS, Lillemoe KD (2004) Risk factors and outcomes in postpancreaticoduodenectomy pancreaticocutaneous fistula. J. Gastrointest. Surg: Official journal of the Society for Surgery of the Alimentary Tract 8: 951-959.

13 Moon HJ, Heo JS, Choi SH, Joh JW, Choi DW, et al. (2005) The efficacy of the prophylactic use of octreotide after a pancreaticoduodenectomy. Yonsei Med J 46:788-793.

14 de Castro SM, Busch OR, van Gulik TM, Obertop H, Gouma DJ (2005) Incidence and management of pancreatic leakage after pancreatoduodenectomy. Br J Surg 92: 1117-1123.

15 Yang YM, Tian XD, Zhuang Y, Wang WM, Wan YL, et al. (2005) Risk factors of pancreatic leakage after pancreaticoduodenectomy. World J Gastroenterol: WJG 11: 2456-2461.

16 Nahm CB, de Ruever PR, Hugh TJ, Pearson A, Gill AJ, et al. (2017) IntraOperative Amylase Concentration in Peri-Pancreatic Fluid Predicts Pancreatic Fistula After Distal Pancreatectomy. J Gastrointest Surg 1-7.

17 Sugimoto M, Takahashi S, Gotohda N, Kato $\mathrm{Y}$, Kinoshita T, et al. (2013) Schematic pancreatic configuration: A risk assessment for postoperative pancreatic fistula after pancreaticoduodenectomy. J Gastrointest Surg 17: 1744-1751.

18 Yamamoto Y, Sakamoto Y, Nara S, Esaki M, Shimada K, et al (2011) A preoperative predictive scoring system for postoperative pancreatic fistula after pancreaticoduodenectomy. World J Surg 35: 2747-2755.

19 Callery MP, Pratt WB, Kent TS, Chaikof EL, Vollmer CM, (2013) A prospectively validated clinical risk score accurately predicts pancreatic fistula after pancreatoduodenectomy. Journal of the American College of Surgeons 216:1-14.

20 Yeo CJ, Cameron JL, Sohn TA, Lillemoe KD, Pitt HA, et al. (1997) Six hundred fifty consecutive pancreaticoduodenectomies in the 1990s: Pathology, complications, and outcomes. Ann Surg 226: 248-257.

21 Büchler MW, Friess H, Wagner M, Kulli C, Wagener V, et al. (2000) Pancreatic fistula after pancreatic head resection. Br J Surg 87: 883-889.

22 Bassi C, Falconi M, Salvia R, Mascetta G, Molinari E, et al. (2001) Management of complications after pancreaticoduodenectomy in a high volume centre: Results on 150 consecutive patients. Dig Surg 18: 453-458.

23 Sarr MG, Pancreatic Surgery G (2003) The potent somatostatin analogue vapreotide does not decrease pancreas-specific complications after elective pancreatectomy: A prospective, multicenter, double-blinded, randomized, placebo-controlled trial. J Am Coll Surg 196: 556-565. 